\title{
Cerebral Vasoreactivity in Parkinson's Disease: A Pilot Study
}

Giuliano Sette $^{1}$, Elisabetta Indelicato ${ }^{1,2}$, Alessandra Fanciulli ${ }^{2}$, Jacob Shofany ${ }^{3}$ and Francesco E. Pontieri ${ }^{13^{*}}$

${ }^{1}$ Department of Neuroscience, Mental Health and Sensory Organs (NESMOS), "Sapienza" University of Rome, Italy

${ }^{2}$ Department of Neurology, Medical University of Innsbruck, Austria

${ }^{3}$ Department of Clinical and Behavioral Neurology, IRCCS "Santa Lucia Foundation", Rome, Italy

\section{Abstract}

Background: Cardiovascular autonomic failure is a frequent non-motor symptom of Parkinson's disease (PD), its key feature being orthostatic hypotension $(\mathrm{OH})$. Recent studies suggest that blood pressure deregulation in PD may be associated with increased cerebrovascular damage. Cerebral vasoreactivity (CVR) refers to the vasodilatory response of cerebral arterioles to elevation of $\mathrm{pCO}_{2}$. Reduced CVR is considered a risk factor for chronic cerebrovascular damage in the setting of hypertension or severe carotid artery stenosis. Here we evaluated CVR in PD patients with or without cardiovascular autonomic failure and age-matched healthy subjects.

Methods: Transcranial doppler ultrasound was applied to measure blood flow velocity in the middle cerebral arteries of $12 \mathrm{PD}$ patients and 11 healthy subjects. CVR was quantified by means of the breath holding index (BHI). OH was identified during a standing test, and in this setting blood flow velocities were likewise recorded.

Results: PD patients displayed significantly lower BHI compared to controls, independently from the presence of $\mathrm{OH}$. Patients with $\mathrm{OH}$ had lower mean arterial blood flow velocities compared to those without $\mathrm{OH}$, both in supine and orthostatic position.

Conclusions: These preliminary results show interactions between cerebral hemodynamics and blood pressure disturbances in $\mathrm{PD}$, that may contribute to chronic cerebrovascular ischemic damage in PD.

\section{Introduction}

Cardiovascular autonomic failure (CAF) is a frequent non-motor symptom of Parkinson's disease (PD), occurring in up to $30 \%$ of cases [1]. The key feature of CAF is orthostatic hypotension $(\mathrm{OH})$, defined as the decrease of systolic blood pressure (BP) $\geq 20 \mathrm{mmHg}$ or diastolic $\mathrm{BP} \geq 10 \mathrm{mHg}$ upon standing or $60^{\circ}$ head-up tilting [2]. This is frequently accompanied by supine and/or nocturnal hypertension, as the result of global alteration of BP control $[3,4]$.

Several research groups suggested the association between BP dysfunctions and cerebrovascular damage in PD [5]. Thus, CAF was coupled with a higher burden of cerebral white matter lesions, i.e. regions of altered parenchymal signal at CT/MRI scan due to chronic cerebrovascular damage. Notably, white matter lesions contribute to neurological disability in PD, in particular by aggravating symptoms scarcely responsive to dopamine replacement therapy such as postural instability and cognitive impairment [6].

Despite the potential relevance of these previous findings, several clues need to be elucidated. Indeed, results in the literature suffer from biases due to the great variability of duration and severity of BP deregulation in PD. Moreover, the pathophysiological mechanisms underlying the association between $\mathrm{BP}$ fluctuations and cerebrovascular damage in PD are still undefined. Eventually, very few data are available on cerebral vasoreactivity (CVR) in PD patients $[7,8]$.

CVR refers to the dilatory response of cerebral arterioles to elevation of $\mathrm{CO}_{2}$ partial pressure [9]. Reduced arteriolar tone leads to increased blood flow velocity in cerebral vessels aimed at "washing out" $\mathrm{CO}_{2}$ from the brain, thus maintaining $\mathrm{pH}$ and cerebral homeostasis. CVR is a chemically-based process initiated within the endothelium, and is viewed as a marker of preserved endothelial functions [10]. CVR may be reduced in individuals suffering from hypertension [10]. Moreover, reduced CVR is an independent predictor for stroke in the setting of severe carotid artery stenosis [11].

\author{
Publication History: \\ Received: November 14, 2017 \\ Accepted: January 11, 2018 \\ Published: January 13, 2018
}

\section{Keywords:}

Breath holding index, Cardiovascular autonomic failure, Cerebral vasoreactivity, Orthostatic hypotension, Parkinson's disease, Transcranial doppler sonography
In outpatient subjects, hypercapnia may be obtained by holding breath, and the subsequent variations in blood flow velocities can be monitored by transcranial doppler sonography of the main cerebral vessels. The percentage increase of blood flow velocity divided by the duration of apnoea, defined as "breath holding index" (BHI), has been widely utilized to estimate CVR (see also "Methods" sections) [9].

To gain further knowledge on the influence of autonomic disturbances on cerebral hemodynamics in PD and to evaluate BHI as a possible marker in this context, here we measured $\mathrm{BHI}$ in $\mathrm{PD}$ patients with and without CAF as compared to healthy subjects.

\section{Materials and Methods}

Twelve subjects diagnosed with PD according to international criteria [12] and 11 healthy subjects were enrolled. All subjects underwent clinical evaluation to exclude major metabolic (e.g., diabetes, dyslipidemia), cardiovascular or neurological co-morbidity, neuroimaging study (CT or MRI) to exclude major strokes, and duplex sonography to exclude significant carotid artery stenosis $(>30 \%)$. The study protocol was approved by the "Santa Lucia Foundation" IRCCS Ethical Committee and conducted in accordance with the Declaration of Helsinki. Each participant signed informed consent prior to enrollment.

To minimize drug influence, we performed all evaluations before the first morning dose of dopaminergic drugs and/or anti-hypertensive "Corresponding Author: Dr. Francesco E. Pontieri, Department of Clinical and Behavioral Neurology, IRCCS Santa Lucia Foundation, Rome, Italy; E-mail: fe.pontieri@gmail.com

Citation: Sette G, Indelicato E, Fanciulli A, Shofany J, Pontieri FE, et al. (2018) Cerebral Vasoreactivity in Parkinson's Disease: A Pilot Study. Int J Clin Res Trials 3: 122. doi: https://doi.org/10.15344/2456-8007/2018/122

Copyright: (c) 2018 Pontieri et al. This is an open-access article distributed under the terms of the Creative Commons Attribution License, which permits unrestricted use, distribution, and reproduction in any medium, provided the original author and source are credited. 
Citation: Sette G, Indelicato E, Fanciulli A, Shofany J, Pontieri FE, et al. (2018) Cerebral Vasoreactivity in Parkinson's Disease: A Pilot Study. Int J Clin Res Trials 3: 122. doi: https://doi.org/10.15344/2456-8007/2018/122

Page 2 of 3

medication. On the examination day subjects were also invited not to drink any beverage containing caffeine, theine or taurine.

To evaluate CVR, hypercapnia was reproduced by holding breath. The subsequent increase in mean blood flow velocity is expressed as a percentage and divided by the seconds of apnoea. The obtained parameter is known as "breath holding index" (BHI) (see below) [8].

$$
\mathrm{BHI}=\left(\frac{\mathrm{V}_{\text {apnoea }}-\mathrm{V}_{\text {basal }}}{\mathrm{V}_{\text {basal }}} \times 100\right) / \text { Time }_{\text {apnoea }}
$$

Variation in blood flow velocities were monitored with transcranial doppler ultrasound. Middle cerebral arteries were bilaterally insonated through the temporal bone window using a headband, and blood flow velocities were continuously recorded after 10 minutes resting in supine position before performing the breath holding. Afterwards, a standing test was performed for $3 \mathrm{~min}$, and mean blood flow velocities were likewise recorded prior and during a further breath holding test.

The presence of $\mathrm{OH}$ in PD patients was evaluated by means of the standing test according to the current international diagnostic criteria [2].

The statistical analysis was performed using SPSS, version 20.0 Normal distribution of data was checked using the Shapiro-Wilk test. Quantitative variables were analyzed by means of unpaired t-test if they showed a normal distribution or Mann-Whitney-U test if not. Fisher's exact test was used to compare categorical variables. P values $<0.05$ were considered statistically significant.

\section{Results}

\section{Comparison between PD patients and controls}

The demographic and clinical data of PD patients and controls are shown in Table 1. The prevalence of hypertension or smoking habit did not differ between PD patients and controls. PD patients displayed significantly higher $\mathrm{BP}$ and heart rate values together with a trend towards lower basal blood flow velocity values. At CVR assessment, $\mathrm{BHI}$ was significantly lower in the PD patients with respect to healthy subjects.

\begin{tabular}{|l|l|l|l|}
\hline & PD Patients & Controls & $p$ value \\
\hline Subjects (Male/Female) & $12(9 / 3)$ & $11(11 / 0)$ & \\
\hline Age (years) & $\begin{array}{l}62 \\
\text { (IQ: 51-77) }\end{array}$ & $\begin{array}{l}59 \\
\text { (IQ: } 48-65)\end{array}$ & 0.347 \\
\hline Disease duration (years) & $5.8 \pm 2.4$ & n.a. & \\
\hline Daily LED (mg) & $600 \pm 200$ & n.a. & \\
\hline Hypertension (n. of cases, $\%)$ & $1(8,3 \%)$ & $3(27,3 \%)$ & 0.261 \\
\hline Smoking (yes/no) & $3(25 \%)$ & $2(18,2 \%)$ & 0.545 \\
\hline Mean BP (mmHg) & $99 \pm 17$ & $86 \pm 12$ & ${ }^{\star} 0.033$ \\
\hline Heart Rate & $80 \pm 15$ & $63 \pm 9$ & ${ }^{\star} 0.002$ \\
\hline $\begin{array}{l}\text { Basal Mean Blood Flow } \\
\text { Velocity (cm/s) }\end{array}$ & $43 \pm 15$ & $52 \pm 8$ & 0.069 \\
\hline BHI & $0.92 \pm 0.30$ & $1.40 \pm 0.44$ & ${ }^{\star} 0.007$ \\
\hline
\end{tabular}

Table 1: Clinical features and hemodynamic parameters of patients and healthy controls (Age displayed as median and interquartile range, the other variables as means \pm SD). LED: L-DOPA equivalent dose calculated according to Tomlinson et al., 2010 [13], n.a.: not applicable, ${ }^{*}$ statistically significant comparison.

\section{Patients intra-group comparison}

During the standing test, $\mathrm{OH}$ was diagnosed in 4/12 PD patients. These patients $\left(\mathrm{OH}^{+}\right)$had comparable disease duration, but they were older and received significantly lower daily amount of dopamine replacement therapy as compared to $\mathrm{OH}^{-}$(see table 2). $\mathrm{OH}^{+}$patients displayed a tendency towards higher supine mean $\mathrm{BP}$ values and significantly lower supine basal blood flow velocities. Nevertheless, in the CVR assessment both groups had similar BHI values. Upon standing, apart from the expected significant differences in BP values, heart rate and blood flow velocity variation did not display significant differences between $\mathrm{OH}^{+}$and $\mathrm{OH}^{-}$patients (Table 2, "Difference standing-supine" section). $\mathrm{OH}^{+}$patients displayed reduced mean blood flow velocity also upon standing comparing to $\mathrm{OH}^{-}$.

\begin{tabular}{|l|l|l|l|}
\hline & OH- & OH+ & p value \\
\hline $\mathrm{N}$ (Male/Female) & $8(5 / 3)$ & $4(4 / 0)$ & \\
\hline Age (years) & $60 \pm 14$ & $70 \pm 13$ & 0.253 \\
\hline Disease duration (years) & $5.9 \pm 2$ & $5.5 \pm 4$ & 0.875 \\
\hline Daily LED (mg) & $693 \pm 169$ & $414 \pm 105$ & ${ }^{\star} 0.006$ \\
\hline Supine & & & \\
\hline Mean BP (mmHg) & $92 \pm 12$ & $115 \pm 16$ & 0.052 \\
\hline Heart Rate & $83 \pm 16$ & $76 \pm 13$ & 0.435 \\
\hline Mean B/F Velocity (cm/s) & $50 \pm 15$ & $34 \pm 8$ & ${ }^{\star} 0.043$ \\
\hline BHI & $0.91 \pm 0.35$ & $0.92 \pm 0.23$ & 0.971 \\
\hline Standing & & & \\
\hline Mean BP (mmHg) & $96 \pm 16$ & $74 \pm 14$ & ${ }^{\star} 0.043$ \\
\hline Heart Rate & $88 \pm 14$ & $89 \pm 7$ & 0.823 \\
\hline Mean B/F Velocity (cm/s) & $46 \pm 16$ & $28 \pm 7$ & ${ }^{\star} 0.025$ \\
\hline BHI & $0.80 \pm 0.36$ & $0.87 \pm 0.19$ & \\
\hline patients with and without OH (values displayed as means $\pm S D)$, \\
\hline \begin{tabular}{l} 
Difference Standing-supine \\
\hline mean BP (mmHg)
\end{tabular} & $4 \pm 7$ & & \\
\hline$\Delta$ Heart rate & $5 \pm 6$ & $8 \pm 4$ & 0.263 \\
\hline$\Delta$ Mean B/F Velocity (cm/s) & $-4 \pm 5$ & $-6 \pm 1$ & 0.223 \\
\hline$\Delta$ BHI & $0.13 \pm 0.24$ & $0.40 \pm 0.18$ & 0.339 \\
\hline
\end{tabular}

\section{Discussion}

The results of this pilot study demonstrate significant difference of CVR between PD patients and controls. These findings are unlikely related to $\mathrm{CAF}$, as $\mathrm{OH}^{+}$and $\mathrm{OH}^{-} \mathrm{PD}$ patients displayed similar performances.

We applied BHI to challenge CVR because of its feasibility in outpatient setting. Furthermore, previous reports showed that such method is equivalent to other standardized tests, such as re-breathing techniques or acetazolamide challenge [9].

To our best knowledge, only one study dealt with BHI evaluation in PD patients previously [7]. Our $\mathrm{OH}^{+}$and $\mathrm{OH}-\mathrm{PD}$ patients displayed similar BHI values as compared to those by Camargo et al. [7]. However, our $\mathrm{OH}^{+}$cases had significantly lower basal mean blood flow velocity with respect to $\mathrm{OH}^{-}$(Table 2). Furthermore, Zamani 
et al. [8] reported a blunted increase in blood flow velocities upon inhalation of CO2-enriched air in 34\% of their PD patients, that was not related to the presence of $\mathrm{OH}$. In that study, however, comparison with control subjects was not performed.

We acknowledge that the small sample size limits the power of our study. However, we believe that the present findings are strengthened by comprehensive pre-test evaluation to exclude the most relevant confounding factors (metabolic disorders, carotid artery stenosis, other causes of cerebrovascular disorders that may influence CVR). Furthermore, differently from previous reports [7], in our study $\mathrm{OH}$ was identified according to the current international guidelines after 3 min standing [2].

CVR is an endothelial-dependent mechanism that may be compromised in several disorders, such as hypertension, carotid artery stenosis and dementia $[10,11]$. The results of this pilot study, together with previous reports $[7,8]$, show altered CVR in PD patients, independently from the presence of CAF. Further, we provide preliminary evidence of lower basal blood flow velocity in $\mathrm{OH}+$ as compared to OH- PD patients while supine and upon standing. These findings, therefore, show reduced CVR compliance in PD patients, that might play a role in the development of chronic cerebrovascular damage facilitated by the presence of cardiovascular autonomic failure. The pathophysiological basis and the prognostic relevance of these preliminary findings, however, remain to be established by future prospective studies on larger cohorts.

\section{Competing Interests}

The Authors declare no conflict of interest with regard to this manuscript.

\section{Acknowldgments}

This was an academic project; no external financial support was assigned.

\section{References}

1. Velseboer DC, de Hann RJ, Wieling W, Goldstein DS, de Bie RM, et al. (2011) Prevalence of orthostatic hypotension in Parkinson's disease: a systematic review and meta-analysis. Parkinsonism Relat Disord 17: 724 729 .

2. Freeman R, Wieling W, Axelrod FB, Benditt DG, Benarroch E, et al. (2011) Consensus Statement on the definition of orthostatic hypotension, neurally mediated syncope and the postural tachycardia syndrome. Clin Autonom Res 21: 69-72.

3. Fanciulli A, Strano S, Colosimo C, Caltagirone C, Spalletta G, et al (2013) The potential prognostic role of cardiovascular autonomic failure in a-synucleinopathies. Eur J Neurol 20: 231-235.

4. Fanciulli A, Goebel G, Ndayisaba JP, et al. (2016) Supine hypertension in Parkinson's disease and multiple system atrophy. Clin Autonom Res 26: 97-105.

5. Indelicato E, Fanciulli A, Poewe W, Antonini A, Pontieri FE, et al. (2015) Cerebral autoregulation and white matter lesions in Parkinson's disease and multiple system atrophy. Parkinsonism Relat Disord 21: 1393-1397.

6. Bohnen NI, Albin RL (2011) White matter lesions in Parkinson disease. Nat Rev Neurol 7: 229-236.

7. Camargo CHF, Martins EA, Lange MC, Hoffmann HA, Luciano JJ, et al (2015) Abnormal cerebrovascular reactivity in patients with Parkinson's disease. Parkinson's Dis 523041.
8. Zamani B, Mehrabani M, Fereshtehnejad SM, Rohani M (2011) Evaluation of cerebral vasomotor reactivity in Parkinson's disease: is there any association with orthostatic hypotension? Clin Neurol Neurosurg 5: 368372.

9. Markus HS, Harrison MJ (1992) Estimation of cerebrovascular reactivity using transcranial doppler, including the use of breath-holding as a vasodilatory stimulus. Stroke 23: 668-673.

10. Lavi S, Gaitini D, Milloul V, Jacob G (2006) Impaired cerebral CO2 vasoreactivity: association with endothelial dysfunction. Am J Physiol Heart Circ Physiol 291: 1856-1861.

11. Reinhard M, Schwarzer G, Briel M, Altamura C, Palazzo P et al. (2014) Cerebrovascular reactivity predicts stroke in high-grade carotid artery disease. Neurology 83: 1424-1431.

12. Gelb DJ, Oliver E, Gilman S (1999) Diagnostic criteria for Parkinson's disease. Arch Neurol 56: 33-39.

13. Tomlinson CL, Stowe R, Patel S, Rick C, Gray R, et al. (2010) Systematic review of levodopa dose equivalency reporting in Parkinson's disease. Mov Disord 15: 2649-2653. 